\title{
THE METRIC ENTROPY OF DIFFEOMORPHISMS
}

\author{
BY F. LEDRAPPIER AND L.-S. YOUNG ${ }^{1}$
}

0. Introduction. Let $M$ be a $C^{\infty}$ compact Riemannian manifold without boundary, let $f$ be a $C^{2}$ diffeomorphism of $M$, and let $m$ be an $f$-invariant Borel probability measure on $M$. The entropy of $f, h_{m}(f)$, measures the amount of randomness with respect to $m$ generated by iterating $f$. In this note we announce some results concerning the relationship between $h_{m}(f)$ and the geometric properties of $f$ and $m$. Our main results are the characterization of measures satisfying Pesin's entropy formula (Theorem 1) and the generalization of this formula to one that applies to all invariant measures (Theorem 2). This generalized formula involves the notion of dimension and leads to other volume estimates (Theorem 3, Corollaries 4 and 5).

1. Preliminaries. By a theorem of Oseledecs [O], for $m$-a.e. $x$ there are numbers $\lambda_{1}(x)>\cdots>\lambda_{r(x)}(x)$ and a decomposition of the tangent space $T_{x} M$ at $x$ into $T_{x} M=E_{1}(x) \oplus \cdots \oplus E_{r(x)}(x)$ such that for every nonzero vector $v \in E_{i}(x),(1 / n) \log \left\|D f_{x}^{n} v\right\| \rightarrow \lambda_{i}(x)$ as $n \rightarrow \pm \infty$.

For $x$ and $i$ with $\lambda_{i}(x)>0$, let

$$
W^{i}(x)=\left\{y \in M: \liminf _{n \rightarrow \infty}-\frac{1}{n} \log d\left(f^{-n} x, f^{-n} y\right) \geq \lambda_{i}(x)\right\} .
$$

Then at a.e. $x, W^{i}(x)$ is an immersed copy of Euclidean space, the tangent space to $W^{i}(x)$ at $x$ being $\bigoplus_{j \leq i} E_{j}(x)[\mathbf{R u}]$. If $u(x)=\max \left\{i: \lambda_{i}(x)>0\right\}$, then $W^{u(x)}(x)$ is called the unstable manifold at $x$.

Recall that if $\xi$ is a measurable partition of $M$, then there is a family of conditional measures $\left\{m_{x}^{\xi}\right\}$ defined at $m$-a.e. $x$ such that the support of $m_{x}^{\xi}$ lies in $\xi(x)$, the element of $\xi$ containing $x$, and $m(A)=\int m_{x}^{\xi}(A) m(d x)$ for every measurable set $A \subset M$. (See [Ro].)

Whenever $W^{i}(x)$ is defined and is an immersed submanifold, the Riemannian structure on $M$ induces a Riemannian structure and, hence, a Riemannian measure on $W^{i}(x)$. We denote this measure by $\mu_{x}^{i}$. A measurable partition $\xi$ is said to be subordinate to $W^{u(\cdot)}$ if for $m$-a.e. $x, \xi(x) \subset W^{u(x)}(x)$ and contains an open neighborhood of $x$ in $W^{u(x)}(x)$. Partitions adapted to $W^{i}$ are defined similarly. We say that $m$ has absolutely continuous conditional measures on unstable manifolds if for every $\xi$ subordinate to $W^{u(\cdot)}, m_{x}^{\xi}$ is absolutely continuous to $\mu_{x}^{u(x)}$ at a.e. $x$.

We omit in the statements of our theorems the standing hypotheses that $f: M \rightarrow M$ is a $C^{2}$ diffeomorphism and $m$ is an $f$-invariant Borel probability measure on $M$.

Received by the editors December 7, 1983.

1980 Mathematics Subject Classification. Primary 58F15.

Key words and phrases. Dimension, entropy, Lyapunov exponents, unstable manifold.

${ }^{1}$ This author is supported by National Science Foundation Grant MCS 8120790. 


\section{Measures satisfying Pesin's formula.}

THEOREM 1. The measure $m$ has absolutely continuous conditional measures on unstable manifolds iff

$$
h_{m}(f)=\int \sum_{i} \lambda_{i}^{+}(x) \operatorname{dim} E_{i}(x) m(d x),
$$

where $a^{+}=\max (a, 0)$.

This formula is called Pesin's formula $[\mathbf{P}]$. See $[\mathbf{L S}]$ for a proof of the forward implication. The (nonuniformly) hyperbolic case of the converse is handled in $[\mathbf{L}]$.

3. A generalized entropy formula. If $m$ is a finite Borel measure on a compact metric space and $B(x, \varepsilon)$ denotes the ball of radius $\varepsilon$ about the point $x$, then we say that $m$ satisfies a volume lemma if

$$
\lim _{\varepsilon \rightarrow 0} \frac{\log m B(x, \varepsilon)}{\log \varepsilon}
$$

exists and is constant $m$-a.e. We refer to this constant, if it exists, as the dimension of the measure $m$, written $\operatorname{dim}(m)$. This definition agrees with various other notions of dimension. (See $[\mathbf{Y}]$.)

We first discuss the ergodic version of our theorem, where $u(x) \equiv u$ a.e.

THEOREM 2. Let $m$ be ergodic. Then there are numbers $\left\{\delta_{i}, i=1, \ldots, u\right\}$, with $0 \leq \delta_{i} \leq \operatorname{dim} E_{i}$, such that for any partition $\xi$ subordinate to $W^{i}$ the conditional measures $m_{x}^{\xi}$ satisfy a volume lemma with $\operatorname{dim}\left(m_{x}^{\xi}\right)=\sum_{j \leq i} \delta_{j}$ and that $h_{m}(f)$ is given by

$$
h_{m}(f)=\sum_{i} \lambda_{i}^{+} \delta_{i}
$$

IDEAS BEHIND THE PROOF. We introduce the notion of entropy along $W^{i}$, which we denote by $h_{i}$. Intuitively, $h_{i}$ measures the amount of randomness along $W^{i}$-leaves. First we show, as in $[\mathbf{Y}]$, that $h_{1}=\delta_{1} \lambda_{1}$. This basic relationship holds whenever the map in question has only one exponent and this exponent is positive. For a.e. $x, W^{i+1}(x)$ is foliated by $W^{i}$-leaves. The map $f: W^{i+1}(x) \rightarrow W^{i+1}(f x)$ behaves like a skew product with respect to these fibers, and its base transformation acts as though its (only) exponent is $\lambda_{i+1}$. Our earlier observation points to the equation $h_{i+1}-h_{i}=\delta_{i+1} \lambda_{i+1}$, where $\delta_{i+1}$ is the "transverse dimension" of $W^{i}$-leaves in $W^{i+1}(x)$. Writing $\operatorname{dim}\left(m^{i}\right)$ for the dimension of the conditional measures of $m$ on $W^{i}$-leaves, we also show that

$$
\operatorname{dim}\left(m^{i+1}\right)=\delta_{i+1}+\operatorname{dim}\left(m^{i}\right) .
$$

Summing these formulas over $i$, we arrive at the conclusion of Theorem 2 .

Without the assumption of ergodicity, Theorem 2 generalizes to the following: 
THEOREM $2^{\prime}$. There are measurable functions $\delta_{i}(x)$ with $0 \leq \delta_{i}(x) \leq$ $\operatorname{dim} E_{i}(x)$ s.t.

$$
h_{m}(f)=\int \sum_{i} \lambda_{i}^{+}(x) \delta_{i}(x) m(d x) .
$$

Note that Ruelle's inequality follows immediately from this formula, and that the numbers $\delta_{i}(x)$ carry the same geometric meaning as before.

4. Volume estimates. We assume ergodicity for the rest of the announcement. Corresponding to each $\lambda_{i}>0$, we have defined a "partial dimension" $\delta_{i}$. For $i$ with $\lambda_{i}<0$ we now define $\delta_{i}$ using $f^{-1}$ and let $\operatorname{dim}\left(m^{+}\right)=$ $\sum_{\left\{i, \lambda_{i}>0\right\}} \delta_{i}, \operatorname{dim}\left(m^{-}\right)=\sum_{\left\{i, \lambda_{i}<0\right\}} \delta_{i}$, and $\operatorname{dim}\left(m^{0}\right)=$ multiplicity of 0 as an exponent.

THEOREM 3. If $m$ is ergodic, then for a.e. $x$,

$$
\limsup _{\varepsilon \rightarrow 0} \frac{\log m B(x, \varepsilon)}{\log \varepsilon} \leq \operatorname{dim}\left(m^{+}\right)+\operatorname{dim}\left(m^{-}\right)+\operatorname{dim}\left(m^{0}\right) .
$$

COROLlaRY 4. Suppose $m$ is ergodic, none of the exponents are zero, and

$$
\limsup _{\varepsilon \rightarrow 0} \frac{\log m B(x, \varepsilon)}{\log \varepsilon}=\operatorname{dim} M
$$

at $m$-a.e. $x$. Then $m$ is absolutely continuous to Riemannian measure on $M$.

This follows immediately from Theorem 3 and the absolute continuity of the stable and unstable foliations. With an obvious modification in its hypotheses, Corollary 4 is also valid for $C^{2}$ flows.

Finally, we state a corollary that partially confirms a conjecture of Yorke, which states that, in some generic sense, the dimension of attractors is given by

$$
\operatorname{Lyap} \operatorname{dim}(m)=\sum_{i<j} \operatorname{dim} E_{i}+\sum_{i<j} \lambda_{i} \operatorname{dim} E_{i} /\left|\lambda_{j}\right|,
$$

where $j$ is the smallest integer such that $\sum_{i \leq j} \lambda_{i} \operatorname{dim} E_{i}<0$, and these exponents are taken with respect to a Bowen-Ruelle measure $m$ [FKYY].

CoRollary 5. For any ergodic measure $m$,

$$
\limsup _{\varepsilon \rightarrow 0} \frac{\log m B(x, \varepsilon)}{\log \varepsilon} \leq \text { Lyap } \operatorname{dim}(m)
$$

at $m$-a.e. $x$. Equality is attained only if $m$ has absolutely continuous conditional measures on unstable manifolds.

\section{REFERENCES}

[FKYY] P. Frederickson, J. Kaplan, E. Yorke and J. Yorke, The Lyapunov dimension of strange attactors (to appear).

[L] F. Ledrappier, Propriétés ergodiques des mesures de Sinai (preprint).

[LS] F. Ledrappier and J. M. Strelyn, $A$ proof of the estimation from below in Pesin entropy formula, Ergodic Theory Dynamical Systems (to appear).

[O] Y. I. Oseledec, A multiplicative ergodic theorem, Lyapunov characteristic numbers for $d y$ namical systems, Trudy Moskov. Mat. Obsc. 19 (1968), 179-210. 
[P] J. Pesin, Characteristic Ljapunov exponents and smooth ergodic theory, Uspekhi. Mat. Nauk 32 (1977), no. 4 (196), 55-112, 287. (Russian)

[Ro] V. A. Rohlin, On the fundamental ideas of measure theory, Amer. Math. Soc. Transl. (2) 10 (1962), pp. 1-54.

[Ru] D. Ruelle, Ergodic theory of differentiable dymamical systems, Inst. Hautes Études Sci. Publ. Math. 50 (1979), 27-58.

[Y] L.-S. Young, Dimension, entropy and Lyapunov exponents, Ergodic Theory Dynamical Systems 2 (1982), 109-124.

Laboratoire de Probabilités, Université Paris VI, 4 Place JussieuTOUR 56, 75230 PARIS, FRANCE

MATHEMATICAL SCIENCES RESEARCH INSTItUTe, BERKELEy, CALIFORNIA 94720

Department of Mathematics, Michigan State University, East LansING, MICHIGAN 48824 